\title{
Prevalencia del segundo canal en la raíz mesiovestibular de los primeros molares maxilares mediante tomografía computarizada de haz de cono
}

\section{Prevalence of a second canal in the mesiobuccal root of maxillary first molars by Cone-Beam Computed Tomography}

\author{
Betancourt P*, Fuentes $\mathrm{R}^{* *}$, Aracena Rojas $\mathrm{S}^{* * *}$, Cantín $\mathrm{M}^{* * * *}$, \\ Navarro Cáceres $\mathrm{P} * * * * *$
}

\section{RESUMEN}

Introducción: La morfología del sistema de canales radiculares es compleja y variable. El primer molar maxilar permanente presenta habitualmente un segundo canal en la raíz mesiobucal (MB2). Su falta de localización y total desbridamiento, es la principal causa de fracaso terapéutico. El objetivo fue determinar la prevalencia in vivo del canal MB2 en la raíz mesiovestibular de primeros molares maxilares mediante tomografía computarizada de haz de cono (TCHC).

Material y método: Se examinaron imágenes TCHC de primeros molares maxilares, izquierdos y derechos, en ambos sexos. Se excluyeron las raíces con canales radiculares tratados endodónticamente o con postes, rehabilitados mediante prótesis fija, calcificación de canales, evidencia de radectomía o cirugía periapical. Fueron seleccionadas 32 imágenes TCHC. En todos los casos, se realizó un estudio sistemático de la raíz mesiobucal a través de cortes en el plano axial.

Resultados: Se observó una prevalencia del $68,75 \%$ del canal MB2. No se encontraron diferencias según sexo o lateralidad. El promedio de edad fue de 25,31 años de edad.

Discusión: La alta prevalencia encontrada del canal MB2, debe ser considerada antes de establecer un tratamiento de endodoncia para lograr un resultado óptimo y evitar el fracaso terapéutico. La TCHC es una herramienta útil para su detección, y presenta alta sensibilidad.

Palabras clave: Primer molar maxilar permanente, canal secundario, raíz mesiobucal, Tomografía computarizada de haz de cono.

\section{SUMMARY}

Introduction: The morphology of the root canal system is complex and variable. The first permanent maxillary molar has usually a second canal in the mesiobuccal root (MB2). Its location and total lack of debridement, is the leading cause of treatment failure. The objective was to determine in vivo the prevalence of MB2 canal in mesiobuccal root of maxillary first molars using Cone-beam computed tomography (CBCT).

* Odontólogo. Alumno de Programa de Magister en Odontología, Facultad de Odontología. Universidad de La Frontera. Temuco, Chile.

** Doctor en Odontología. Profesor Asociado. Departamento Integral Adultos, Facultad de Odontología. Universidad de La Frontera. Temuco, Chile.

*** Especialista en Endodoncia. Profesor Asistente. Departamento Integral Adultos, Facultad de Odontología. Universidad de La Frontera. Temuco, Chile.

**** Magíster en Morfología. Programa de Doctorado en Ciencias Morfológicas. Profesor Asistente. Departamento Integral Adultos, Facultad de Odontología. Universidad de La Frontera. Temuco, Chile.

***** Departamento de Matemáticas y Estadística, Universidad de la Frontera. Temuco, Chile. 
Methods: CBCT images of first maxillary molars, left and right, in both sexes, were reviewed. We excluded roots endodontically treated or with posts, rehabilitated with a fixed prosthesis, calcified canals, evidence of root resection or pericapical surgery. 32 first molars were selected. In all cases, we performed a systematic study of the mesiobuccal root through cuts in the axial plane.

Results: There was a prevalence of $68.75 \%$ of MB2 canal. There were no differences by sex or laterality. The average age was 25.31 years old.

Discussion: The high prevalence found MB2 canal should be considered before establishing a root canal treatment to achieve optimal results and avoid treatment failure. The CBCT is a useful tool for detection, and has high sensitivity.

Key words: First permanent maxillary molar, second canal, mesiobuccal root, Cone-beam computed tomography.

Fecha de recepción: 4 de septiembre de 2012.

Aceptado para publicación: 27 de septiembre de 2012 .

Betancourt P, Fuentes R, Aracena Rojas S, Cantín M, Navarro Cáceres P. Prevalencia del segundo canal en la raíz mesiovestibular de los primeros molares maxilares mediante tomografía computarizada de haz de cono. Av. Odontoestomatol 2013; 29 (1): 31-36.

\section{INTRODUCCIÓN}

El éxito del tratamiento endodóntico requiere de un profundo conocimiento de la anatomía y disposición de el sistema de canales radiculares, de modo que se alcance un correcto acceso, limpieza, instrumentación biomecánica y obturación radicular (1).

La morfología del sistema de canales radiculares es compleja y variable, especialmente en los dientes multirradiculares. El primer molar maxilar permanente es el diente que presenta la mayor variación (2), así como la más alta tasa de fracaso ente el de tratamiento endodóntico (3). Clásicamente, su anatomía radicular describe la presencia de tres raíces y tres canales. Sin embargo, diversos estudios señalan una incidencia sobre el $50 \%$ de un segundo canal en la raíz mesiovestibular (MB2) $(1,2,4)$. La falta de localización y total desbridamiento del MB2 es la principal causa de fracaso terapéutico $(4,5)$.

Estudios in vitro sobre el MB2 reportan una tasa de detección del $80 \%$, mientras que in vivo comúnmente sólo alcanza al $40 \%$ (3). Los métodos más utilizados para evaluar la anatomía de los canales radiculares y localizar el MB2 clínicamente son la microscopía y la radiografía convencional. Sin embargo el acceso a un equipo microscópico, y la de- finición y detalle que entrega la radiografía convencional son limitaciones importantes.

La reciente incorporación de la tomografía computarizada de haz de cono (TCHC) ha permitido visualizar estructuras anatómicas de difícil acceso, siendo una valiosa ayuda al suministro de información adicional para el diagnóstico y tratamiento (6), con una menor dosis de radiación que la TC convencional, y mayor precisión que las radiografías digitales para estudiar los sistemas de canales radiculares (7). La correcta identificación del MB2 in vivo es un desafío constante para el diagnóstico, tratamiento y éxito de la terapia endodóntica, y su prevalencia se ha asociado a factores como el origen étnico (4), la edad (8) y el sexo (9).

El objetivo de este estudio, fue describir in vivo la prevalencia del canal MB2 en las raíces mesiovestibulares de primeros molares maxilares permanentes, mediante el estudio de imágenes TCHC.

\section{MATERIAL Y MÉTODO}

Se examinaron imágenes TCHC de primeros molares maxilares, obtenidas desde el Servicio de Imagenología de la Facultad de Odontología de la Universidad de La Frontera, Temuco, Chile, en pacientes 
de ambos sexos entre los meses de noviembre del año 2011 hasta abril del año 2012.

Los exámenes imagenológicos fueron tomados como parte del examen, diagnóstico y planificación de tratamiento. La obtención de imágenes se realizó mediante el equipo CBCT modelo Pax Zenith, marca Vatech (Korea 2011), utilizando 90 kV y 120 mA; FOV $8 \times 6$ $\mathrm{cm}$, tamaño voxel $0,12 \mathrm{~mm}$. Fueron seleccionadas las imágenes en las que se observó la presencia del primer molar superior, y que cumplieran los criterios de inclusión: edad entre 10 y 75 años, y formación radicular completa. Se excluyeron los molares con canales radiculares con relleno endodóntico o postes, rehabilitados mediante prótesis fija, calcificación de canales, evidencia de radectomía o cirugía periapical. Se obtuvieron $32 \mathrm{TCHC}$ de primeros molares permanentes, 16 de cada lado, las que fueron analizadas mediante software Ez 3D 2009, con cortes de 0,5 mm de intervalo y $1 \mathrm{~mm}$ de grosor en el plano axial, identificándose la presencia del canal MB2 sistemáticamente desde el piso de la cámara pulpar hasta la zona apical en todas las muestras. Para su visualización se utilizó pantalla LG LED Modelo 42LE4300-SA. Todas las imágenes fueron analizadas por 2 examinadores especialistas previo entrenamiento y consenso.

Los resultados obtenidos fueron analizados mediante estadística descriptiva, estableciéndose la relación entre la presencia del MB2 según sexo, edad y posición en el maxilar (derecha o izquierda).

\section{RESULTADOS}

La prevalencia del $\mathrm{MB} 2$ en los primeros molares maxilares fue del $68,75 \%$ en los casos analizados. De acuerdo a las características morfológicas en los 22 casos donde se encontró el MB2, fueron paralelos y de menor diámetro que el canal mesiobucal
(Figura 1). La distribución porcentual del canal MB2 según sexo fue homogénea entre hombres y mujeres $(50 \%)$. En cuanto a la lateralidad, no hubo diferencia según el lado estudiado, $50 \%$ en el molar izquierdo y $50 \%$ en molar derecho.

El promedio de edad de la muestra fue 25,3 años. Al analizar los datos que asocian la presencia del MB2 con la edad a través de la prueba t de Student para diferencias de medias, se obtuvo un valor $p=0,661$, lo cual indica que no existen diferencias estadísticamente significativas (Tabla 1).

Según el test exacto de Fisher, no se observaron diferencias significativas al analizar la relación entre presencia del MB2 según sexo $(\mathrm{p}=1,00)$ y lateralidad $(p=1,00)($ Tablas 2 y 3$)$.

\section{DISCUSIÓN}

La determinación de la condición anatómica de la raíz mesiobucal de los primeros molares maxilares

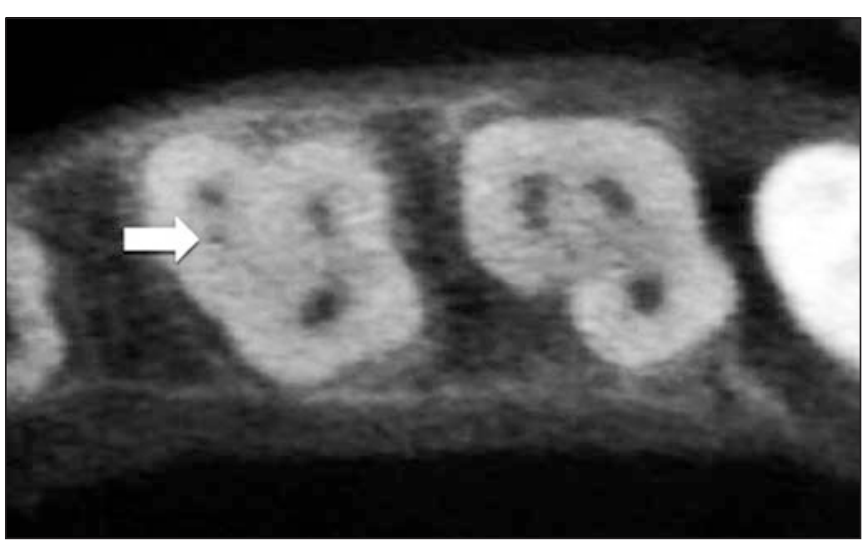

Fig. 1. Visión corte axial de TCHC del primer molar maxilar permanente. Se observa la raíz mesiobucal. La flecha blanca indica la localización del canal mesiobucal accesorio (MB2) de menor diámetro, paralelo canal mesiobucal.

\section{TABLA 1.- PROMEDIO DE EDAD ASOCIADO A LA PRESENCIA DEL CANAL MB2 EN MUESTRA POBLACIÓN-ESPECÍFICA DE TEMUCO, CHILE}

\begin{tabular}{|l|c|c|c|c|c|c|}
\hline MB2 & & $\mathbf{n}$ & Media & Desviación & Error tip. de la media & P \\
\hline \multirow{2}{*}{ Edad } & Ausencia & 10 & 25,4000 & 11,08753 & 3,50619 & 0,661 \\
\cline { 2 - 6 } & Presencia & 22 & 25,3182 & 11,65940 & 2,48579 & \\
\hline
\end{tabular}


TABLA 2.- PREVALENCIA DEL CANAL MB2 SEGÚN SEXO EN MUESTRA-POBLACIÓN ESPECÍFICA DE TEMUCO, CHILE

\begin{tabular}{|l|rr|ll|ll|c|}
\hline \multirow{2}{*}{ Sexo } & \multicolumn{4}{|c|}{ MB2 } & \multirow{2}{*}{ Total } & P \\
\cline { 2 - 6 } & Ausencia & Presencia & & & \\
\hline Femenino & 5 & $(50 \%)$ & 11 & $(50 \%)$ & 16 & $(50 \%)$ & 1,00 \\
\hline Masculino & 5 & $(50 \%)$ & 11 & $(50 \%)$ & 16 & $(50 \%)$ & \\
\hline Total & $10(100 \%)$ & $22(100 \%)$ & 32 & $(100 \%)$ & \\
\hline
\end{tabular}

TABLA 3.- DISTRIBUCIÓN PORCENTUAL DE PREVALENCIA DEL CANAL MB2 SEGÚN LATERALIDAD EN MUESTRA-POBLACIÓN ESPECÍFICA DE TEMUCO, CHILE

\begin{tabular}{|c|c|c|c|c|}
\hline \multirow{2}{*}{ Lado } & \multicolumn{2}{|c|}{ MB2 } & \multirow{2}{*}{ Total } & \multirow{2}{*}{$\mathrm{p}$} \\
\hline & Ausencia & Presencia & & \\
\hline Derecho & $5 \quad(50 \%)$ & $11 \quad(50 \%)$ & $16 \quad(50 \%)$ & \multirow[t]{2}{*}{1,00} \\
\hline Izquierdo & $5 \quad(50 \%)$ & $11 \quad(50 \%)$ & $16 \quad(50 \%)$ & \\
\hline Total & $10(100 \%)$ & $22(100 \%)$ & $32(100 \%)$ & \\
\hline
\end{tabular}

previo al tratamiento endodóntico es difícil. La aparición del sistema TCHC ha facilitado la localización y manejo de los canales radiculares accesorios, especialmente del canal MB2 (10).

En este estudio encontramos una prevalencia de $68,75 \%$ del canal MB2 identificado a través del sistema TCHC in vivo, lo que representa una alta frecuencia de esta variación anatómica. Estos resultados concuerdan con el $71,8 \%$ reportado por Lee y cols. (11), el $63,93 \%$ por Rathi y cols. (12), y son mayores al $54 \%$ reportado por Abuabuara y cols. (10) y $50,40 \%$ por Zheng y cols. (2). Baratto y cols. (13), encontraron en el primer molar maxilar mayoritariamente 3 canales $(59,25 \%)$, seguido por 4 canales (37,05\%), de los cuales el 95,45\% fue MB2. Todos estos estudios in vivo realizados por TCHC sobre diferentes poblaciones; $y$ fueron superiores a los reportados en estudios in vitro utilizando TCHC. Blattner y cols. (14) reportaron en dientes extraídos una frecuencia del 57,9\%. De acuerdo con una revi- sión de la literatura realizada por Cleghorn y cols. (1) entre estudios de laboratorio (in vitro), estudios clínicos (in vivo) y reportes de caso, se describe una incidencia del canal MB2 en un 56,8\%.

Estas observaciones contrastan con lo expuesto por Somma y cols. (15), quienes a través del uso de microtomografía computarizada en dientes extraídos, encontraron una frecuencia del $80 \%$, porcentaje superior al de este estudio y a los reportes de la literatura. Esto puede explicase por la alta sensibilidad del examen. Sin embargo, Michetti y cols. (16) validaron la utilización del TCHC para explorar la anatomía de los canales radiculares comparándola con cortes histológicos, observando una fuerte correlación entre los datos adquiridos a través de las secciones TCHC e histológicas. Por esto, probablemente los resultados controvertidos puedan deberse a otros factores como el tamaño de la muestra o la población estudiada.

Probablemente, como en otras condiciones anatómicas, existe un factor de tipo población-específico implicado en la frecuencia del MB2. Los resultados encontrados en nuestra población, se acercan a los observados en población Koreana $(71,8 \%)(11)$, Tailandesa (68,5\%) (17), Inglesa 65\% (18) e India un $(63,93 \%)(12)$, son superiores a lo observado en población de Brasil (54\%) (10) y China (52\%) (3) y $(50,40 \%)$ (2) y de Kuwait (42\%) (19); mientras que es bajo al ser comparada con la población de Italia $(80 \%)$ (15) y Turquía $(93,5 \%)(9)$.

Otros métodos in vitro han sido utilizados para estudiar la frecuencia del MB2, mostrando una frecuencia similar. Alavi y cols. (18) reportaron un $65 \%$ mediante la clásica técnica de aclaración en dientes extraídos, mientras que Baratto y cols. (13) y Alaçam y cols. (20) al estudiar la detección del canal MB2 mediante microscopía alcanzaron un $67,14 \%$ (del cual un 92,85\% fue MB2), y $67 \%$, respectivamente, y al combinar el uso de ultrasonido con microscopio, aumentó a un $74 \%$ la detección. Sin embargo, al utilizar estos métodos de manera in vivo, alcanzaron porcentajes inferiores, $53,26 \%$ y $62 \%$ respectivamente.

Interesantes son los resultados obtenidos por Abuabuara y cols. (19), quienes realizaron un estudio de prevalencia de canal MB2 a través de radiografías periapicales, las cuales son las más utilizadas 
durante los procedimientos de endodoncia, encontrando una frecuencia sólo del $8 \%$, porcentaje muy inferior a lo reportado en este estudio por TCHC. La $\mathrm{TCHC}$ ofrece resultados superiores en comparación con otros métodos como la radiografía dental digital, microscopía y la observación a simple vista (21). Con éste, se pueden obtener reconstrucciones multiplanares y tridimensionales, además, su radiación es baja y se limita únicamente a la zona anatómica que se está investigando.

Al relacionar la presencia del canal MB2 según la edad, encontramos que el promedio de edad donde se encuentra la más alta frecuencia es a los 25,31 años. Este resultado fue similar al de Zheng y cols. (2) quienes muestran una mayor frecuencia en pacientes entre los 20 y 30 años. Esos resultados difieren a los expuestos por Rathi y cols. (12) donde el promedio de edad de más alta frecuencia del canal MB2 es entre los 51-60 años (29,50\%), seguidos por el grupo de 31-40 y 21-30 años, ambos con 19,67\%. El promedio de edad encontrado en este estudio es menor que el reportado por Lee y cols. (11), el cual se encuentra entre los 30 y 49 años. Estas diferencias pueden deberse al tamaño muestral.

No se encontraron diferencias en cuanto al sexo o su ubicación en el maxilar, observando una distribución homogénea, similar a lo reportado por Zheng y cols. (2), con una distribución del $50 \%$ en mujeres y $54,27 \%$ en hombres, y de un $49,82 \%$ en el lado derecho y $54,13 \%$ en el izquierdo. Lee y cols. (11) reportan una distribución porcentual mayor en hombres $(73,3 \%)$ y mujeres $(69,9 \%)$, y según el lado, $71,7 \%$ en el maxilar derecho y $72 \%$ en el izquierdo. Esto, podría también explicarse por un factor población-específico.

Es fundamental tener en cuenta los cambios en la morfología del sistema de canales radiculares en los dientes que requieren tratamiento endodóntico u otros procedimientos (22), donde se debe realizar un cuidadoso examen clínico y radiológico (1).

\section{CONCLUSIÓN}

Debido a la alta frecuencia de canal MB2 encontrada, es necesario lograr un adecuado acceso endo- dóntico que permita una visualización correcta, y utilizar herramientas como el microscopio o ultrasonido para la detección del canal MB2 en la práctica clínica diaria. Sin embargo, cuando aún se producen problemas en la localización de MB2 en los molares maxilares, es conveniente tener en cuenta la TCHC como método diagnóstico auxiliar antes de establecer un tratamiento de endodoncia para lograr un resultado óptimo y evitar el fracaso terapéutico.

\section{BIBLIOGRAFÍA}

1. Cleghorn B, Christie W, Dong C. Root and root canal morphology of the human permanent maxillary first molar: a literature review. J Endod 2006;32:813-21.

2. Zheng Q, Wang Y, Zhou X, Wang Q, Zheng G, Huang D. A Cone-Beam Computed Tomography Study of Maxillary First Permanent Molar Root and Canal Morphology in a Chinese Population. J Endod 2010;36:1480-4.

3. Zhang R, Yang H, Yu X, Wang H, Hu T, Dummer $\mathrm{PMH}$. Use of CBCT to identify the morphology of maxillary permanent molar teeth in a Chinese subpopulation. Int Endod J 2011;44:162-9.

4. Weine FS, Hayami S, Hata G, Toda T. Canal configuration of the mesiobuccal root of the maxillary first molar of a Japanese subpopulation. Int Endod J 1999;32:79-87.

5. Kontakiotis E, Tzanetakis G. Four canals in the mesial root of a mandibularm first molar. A case report under the operating microscope. Aust Endod J 2007;33:84-8.

6. Patel S, Kanagasingam S, Mannocci F. Cone beam computed tomography (CBCT) in endodontics. Dent Update 2010;37:373-9.

7. Neelakantan P, Subbarao C, Ahuja R, Subbarao C, Gutmann J. Cone-Beam Computed Tomography Study of Root and Canal Morphology of Maxillary First and Second Molars in an Indian Population. J Endod 2010;36:1622-7. 
8. Neaverth EJ, Kotler LM, Kaltenbach RF. Clinical investigation (in vivo) of endodontically treated maxillary first molars. J Endod 1987;13:506-12.

9. Sert S, Bayirli GS. Evaluation of the root canal configurations of the mandibular and maxillary permanent teeth by gender in the Turkish population. J Endod 2004;30:391-8.

10. Abuabara A, Baratto-Filho F, Aguiar Anele J, Leonardi DP, Sousa-Neto MD. Efficacy of clinical and radiological methods to identify second mesiobuccal canals in maxillary first molars. Acta Odontol Scand 2012; Doi 10.3109/ 00016357.2011 .654262

11. Lee J, Kim K, Lee J, Park W, Jeong J, Lee Y, Gu Y, Chang S, Son W, Lee W, Baek S, Bae K, Kum K. Mesiobuccal root canal anatomy of Korean maxillary first and second molars by cone-beam computed tomography Oral Surg Oral Med Oral Pathol Oral Radiol Endod 2011;111:785-91.

12. Rathi S, Patil J, Jaju PP. Clinical Study Detection of Mesiobuccal Canal in Maxillary Molars and Distolingual Canal in Mandibular Molars by Dental CT: A Retrospective Study of 100 Cases. Int J Dent 2010; 2010:291276.

13. Baratto F, Zaitter S, Aihara G, Alves E, Abuabara A, María G. Analysis of the internal anatomy of maxillary first molars by using different methods. J Endod 2009;35:337-42.

14. Blattner T; George N, Lee C, Kumar V, Yelton C. Efficacy of Cone-Beam Computed Tomography as a Modality to Accurately Identify the Presence of Second Mesiobuccal Canals in Maxillary First and Second Molars: A Pilot Study. J Endod 2010;36:867-70.

15. Somma, F, Leoni D, Plotino D, Grande NM, Plasschaert A. Root canal morphology of the mesiobuccal root of maxillary first molars: a microcomputed tomographic analysis. Int Endod J 2008;42:165-74.
16. Michetti J, Maret D, Mallet JP, Diemer F. Validation of cone beam computed tomography as a tool to explore root canal anatomy. J Endod 2010;36:1187-90.

17. Peeters H, Suardita K, Setijanto D. Prevalence of a second canal in the mesiobuccal root of permanent maxillary first molars from an Indonesian population. J Oral Sci 2011;53:48994.

18. Alavi A, Opasanon A, Ng YL, Gulavibala K. Root and canal morphology of thai maxillary molars. Int Endod J 2002; 35:478-85.

19. Pattanshetti N, Gaidhane M, Kandari A. Root and canal morphology of the mesiobuccal and distal roots of permanent first molars in a Kuwait population - a clinical study. Int Endod J 2008; 41:755-62.

20. Alaçam T, Cemal AC, Genç Ö, Kayaoglu G. Second mesiobuccal canal detection in maxillary first molars using microscopy and ultrasonics. Aust Endod J 2008;34:106-9.

21. Hosoya N, Yoshida T, lino F, Arai T, Mishima A, Kobayashi K. Detection of a secondary mesiobuccal canal in maxillary first molar: A comparative study. J Conserv Dent 2012;15:127-31.

22. Oporto G, Fuentes R, Soto C. Variaciones anatómicas radiculares y sistema de canales. Int $\mathrm{J}$ Morphol 2010;28:945-50.

\section{CORRESPONDENCIA}

Ramón Fuentes Fernández

Departamento Integral Adultos

Facultad de Odontología

Universidad de La Frontera

Manuel Montt, 112

Temuco, Chile

Correo electrónico: rfuentes@ufro.cl 